\title{
A dynamic estimation scheme of specific growth rates of bacteria for an anaerobic wastewater treatment process
}

\author{
S. Diop ${ }^{1}$, J. P. Steyer ${ }^{2}$ and I. Simeonov ${ }^{3}$
}

\begin{abstract}
The paper proposes an observability analysis and estimation schemes for specific growth rates for an anaerobic wastewater treatment process. A 2stage model of 6 dynamic states is assumed, describing the acidogenesis and methanogenesis of two different populations of microorganisms (acidogenic and methanogenic), and the evaluation of the total carbon dioxide production including the soluble part. The main result is that the specific growth rates of the two populations of bacteria can be stability estimated only from easily measured quantities - the dilution rate and the flow rates of methane and carbon dioxide in the biogas.
\end{abstract}

\section{INTRODUCTION}

Before it may be discharged in natural water systems the chemical oxygen demand (COD: amount of oxygen needed to consume the organic and inorganic materials) of municipal or industrial wastewater often needs to be highly reduced. Otherwise, this COD amount of dissolved oxygen will be consumed by microorganisms leading to the mortality of aquatic organisms. Among the two usual processes used to reduce COD, aerobic and anaerobic wastewater treatments, the latter advantageously does not require the energy necessary for aeration, and yields methane as a by-product so as its energetic balance is rather positive. Anaerobic wastewater treatment is thus subject of many studies since decades, in particular, as a domain of application of system theory results. The dynamics of this process are the ones of standard anaerobic digestion, and depend on the type of organic matters contained in the wastewater. The specific model that is studied in this work was thoroughly developed in [3] for raw industrial wine distillery vinasses obtained from local wineries in the area of Narbonne, France. It is a 2-stage model of 6 dynamic states, describing the acidogenesis and methanogenesis of two different populations of microorganisms (acidogenic and methanogenic), and the evaluation of the total carbon dioxide production including the soluble part. It has been subject to many works, see for instance [1, 2, 7]. The model is based on mass balance arguments involving abstract quantities

\footnotetext{
${ }^{1} \mathrm{~S}$. Diop is with the CNRS, Laboratoire des Signaux et Systèmes, Supélec, Plateau de Moulon, 91192 Gif sur Yvette cedex, France, diop@lss.supelec.fr

${ }^{2}$ J. P. Steyer is with the Laboratoire de Biotechnologie de l'Environnement, INRA, Avenue des Etangs, Narbonne 11100, France, steyer@supagro.inra.fr

${ }^{3}$ I. Simeonov is with the Institute of Microbiology, Bulgarian Academy of Sciences, Acad. G. Bonchev St., Block 26, Sofia 1113, Bulgaria, issim@microbio.bas.bg
}

named specific growth rates which are complex unknown functions of many other quantities and parameters influencing the dynamics of the process, and describe the kinetics of the process. Empirical modeling which are time consuming and difficult to identify and subject to change with the type of wastes are often invoked to express these specific growth rates. The contribution of the present work is to propose estimation schemes of specific growth rates from supposedly easily measured quantities such as the dilution rate and the flow rates of methane and carbon dioxide in the biogas. In $[1$, 2] estimation of substrate and bacteria concentrations has been considered, without having recourse to specific growth rates [2], and by treating specific growth rates as uncertainties with known bounds [1, 7]. The main point of the present work, compared to the previous ones, is to provide a systematic way to obtain the proposed estimation schemes. The method is part of a general approach of observation problems using tools from differential algebraic geometry [5].

The paper is organized as following. The next section is devoted to the description of the model of the specific anaerobic digestion process. Then the observability of the two specific growth rates is analyzed in the light of the differential algebraic approach. Finally estimation schemes are detailed for most of the quantities involved in the model. Illustrative simulations as well as confrontation to experimental data are postponed to future versions of this paper.

\section{THE PROCESS MODEL}

The following model of wastewater treatment of raw industrial wine distillery vinasses has been thoroughly developed in [3].

$$
\begin{aligned}
& \dot{S}_{1}=D S_{1 \mathrm{in}}-D S_{1}-k_{1} \mu_{1} X_{1} \\
& \dot{X}_{1}=\mu_{1} X_{1}-\alpha D X_{1}, \\
& \dot{S}_{2}=D S_{2 \mathrm{in}}-D S_{2}+k_{2} \mu_{1} X_{1}-k_{3} \mu_{2} X_{2}, \\
& \dot{X}_{2}=\mu_{2} X_{2}-\alpha D X_{2}, \\
& q_{\mathrm{M}}=k_{6} \mu_{2} X_{2}, \\
& q_{\mathrm{C}}=k_{\mathrm{L}} \mathrm{a}\left(C+S_{2}-Z-K_{\mathrm{H}} P_{\mathrm{C}}\right), \\
& \dot{C}=D C_{\text {in }}-D C-q_{\mathrm{C}}+k_{4} \mu_{1} X_{1}+k_{5} \mu_{2} X_{2}, \\
& \dot{Z}=D Z_{\text {in }}-D Z,
\end{aligned}
$$




$$
\begin{aligned}
& P_{\mathrm{C}}=\frac{\phi-\sqrt{\phi^{2}-4 K_{\mathrm{H}} P_{\mathrm{T}}\left(C+S_{2}-Z\right)}}{2 K_{\mathrm{H}}}, \\
& \phi=C+S_{2}-Z+K_{\mathrm{H}} P_{\mathrm{T}}+\frac{k_{6}}{k_{\mathrm{L}} \mathrm{a}} \mu_{2} X_{2}, \\
& \mathrm{pH}=-\log _{10}\left(K_{\mathrm{b}} \frac{C-Z+S_{2}}{Z-S_{2}}\right),
\end{aligned}
$$

\begin{tabular}{|c|c|}
\hline$D$ & dilution rate $\left(\mathrm{d}^{-1}\right)$ \\
\hline$S_{1}, S_{1 \text { in }}$ & organic substrate concentration $(\mathrm{mmol} / \mathrm{L})$ \\
\hline$X_{1}$ & acidogenic bacteria concentration $(\mathrm{g} / \mathrm{L})$ \\
\hline$\mu_{1}$ & specific growth rate of acidogenic bacteria $\left(\mathrm{d}^{-1}\right)$ \\
\hline$S_{2}, S_{2 \text { in }}$ & volatile fatty acids concentration $(\mathrm{mmol} / \mathrm{L})$ \\
\hline$X_{2}$ & methanogenic bacteria concentration $(\mathrm{g} / \mathrm{L})$ \\
\hline$\mu_{2}$ & specific growth rate of methanogenic bacteria $\left(\mathrm{d}^{-1}\right)$ \\
\hline$q_{\mathrm{C}}$ & $\mathrm{CO}_{2}$ flow rate $(\mathrm{mmol} / \mathrm{L}$ per $\mathrm{d})$ \\
\hline$q_{\mathrm{M}}$ & $\mathrm{CH}_{4}$ flow rate $(\mathrm{mmol} / \mathrm{L}$ per $\mathrm{d})$ \\
\hline$C, C_{\mathrm{in}}$ & total inorganic carbon concentration $(\mathrm{mmol} / \mathrm{L})$ \\
\hline$Z, Z_{\text {in }}$ & total alkalinity $(\mathrm{mmol} / \mathrm{L})$ \\
\hline$P_{\mathrm{C}}$ & $\mathrm{CO}_{2}$ partial pressure (atm) \\
\hline$P_{\mathrm{T}}$ & total pressure $(\mathrm{atm})$ \\
\hline$\alpha$ & fraction of bacteria in the liquid phase \\
\hline$k_{1}$ & yield for substrate degradation \\
\hline$k_{2}$ & yield for VFA production ( $\mathrm{mmol} / \mathrm{g})$ \\
\hline$k_{3}$ & yield for VFA consumption $(\mathrm{mmol} / \mathrm{g})$ \\
\hline$k_{4}$ & yield for $\mathrm{CO}_{2}$ production $(\mathrm{mmol} / \mathrm{g})$ \\
\hline$k_{5}$ & yield for $\mathrm{CO}_{2}$ production $(\mathrm{mmol} / \mathrm{g})$ \\
\hline$k_{6}$ & yield for $\mathrm{CH}_{4}$ production $(\mathrm{mmol} / \mathrm{g})$ \\
\hline$k_{\mathrm{a}}, k_{\mathrm{b}}$ & equilibrium constants (mol/L) \\
\hline$K_{\mathrm{H}}$ & Henry's constant (mmol/L per atm) \\
\hline$k_{\mathrm{L}} \mathrm{a}$ & liquid-gas transfer constant $\left(\mathrm{d}^{-1}\right)$ \\
\hline
\end{tabular}

where the nomenclature is given in the following table.

\section{TABLE I: Nomenclature}

\section{OBSERVABILITY ANALYSIS}

The observability analysis that is performed here follows from the approach described in $[5,6]$. In order to fit in the present differential algebraic approach the equation giving $P_{\mathrm{C}}$ will be replaced by the following one

$$
\left(\phi-2 K_{\mathrm{H}} P_{\mathrm{C}}\right)^{2}=\phi^{2}-4 K_{\mathrm{H}} P_{\mathrm{T}}\left(C+S_{2}-Z\right)
$$

keeping in mind the implicitly supposed inequality

$$
\psi=\phi^{2}-4 K_{\mathrm{H}} P_{\mathrm{T}}\left(C+S_{2}-Z\right) \geq 0
$$

and the expression of $\mathrm{pH}$ will be replaced by

$$
\mathrm{pHe}\left(Z-S_{2}\right)=K_{\mathrm{b}}\left(C-Z+S_{2}\right)
$$

where

$$
\mathrm{pHe}=10^{-\mathrm{pH}}
$$

and

$$
\xi=\frac{C-Z+S_{2}}{Z-S_{2}}>0
$$

The wastewater treatment model then becomes

$$
\left\{\begin{array}{l}
\dot{S}_{1}=D\left(S_{1 \text { in }}-S_{1}\right)-k_{1} \mu_{1} X_{1}, \\
\dot{X}_{1}=\left(\mu_{1}-\alpha D\right) X_{1}, \\
\dot{S}_{2}=D\left(S_{2 \text { in }}-S_{2}\right)+k_{2} \mu_{1} X_{1}-k_{3} \mu_{2} X_{2}, \\
\dot{X}_{2}=\left(\mu_{2}-\alpha D\right) X_{2}, \\
\dot{C}=D\left(C_{\text {in }}-C\right)-q_{\mathrm{C}}+k_{4} \mu_{1} X_{1}+k_{5} \mu_{2} X_{2}, \\
\dot{Z}=D\left(Z_{\text {in }}-Z\right), \\
q_{\mathrm{M}}=k_{6} \mu_{2} X_{2}, \\
q_{\mathrm{C}}=k_{\mathrm{L}} \mathrm{a}\left(C+S_{2}-Z-K_{\mathrm{H}} P_{\mathrm{C}}\right), \\
\left(\phi-2 K_{\mathrm{H}} P_{\mathrm{C}}\right)^{2}=\phi^{2}-4 K_{\mathrm{H}} P_{\mathrm{T}}\left(C+S_{2}-Z\right), \\
k_{\mathrm{L}} \mathrm{a} \phi=k_{\mathrm{L}} \mathrm{a} C+k_{\mathrm{L}} \mathrm{a} S_{2}-k_{\mathrm{L}} \mathrm{a} Z+k_{\mathrm{L}} \mathrm{a} K_{\mathrm{H}} P_{\mathrm{T}} \\
\quad+k_{6} \mu_{2} X_{2}, \\
\operatorname{pHe}\left(Z-S_{2}\right)=K_{\mathrm{b}}\left(C-Z+S_{2}\right) .
\end{array}\right.
$$

Online measurements which are supposed to be available for this study, according to [3], are $D, Z_{\text {in }}, S_{1 \mathrm{in}}, S_{2 \mathrm{in}}, C_{\mathrm{in}}$, $q_{\mathrm{M}}, q_{\mathrm{C}}$ and $\mathrm{pH}$. Observability of $\mu_{1}$ and $\mu_{2}$ with respect to these online data is thus first examined.

Technically, this consists of the computation of the characteristic set [5] of the differential polynomial ideal generated by equations in (1) for the following ranking

$$
\begin{aligned}
\{ & \\
& \left\{\alpha, k_{1}, k_{2}, k_{3}, k_{4}, k_{5}, k_{6}, k_{\mathrm{L}} \mathrm{a}, K_{\mathrm{b}}, K_{\mathrm{H}}, P_{\mathrm{T}}\right\}, \\
& \left\{D, Z_{\text {in }}, S_{1 \text { in }}, S_{2 \mathrm{in}}, C_{\text {in }}\right\}, \\
& \left\{q_{\mathrm{M}}, q_{\mathrm{C}}, \mathrm{pHe}\right\} \\
& \left\{\mu_{1}, \mu_{2}\right\} \\
& \left\{X_{1}, X_{2}, Z, S_{1}, S_{2}, C, \phi, \psi, \xi, P_{\mathrm{C}}\right\} \\
\} . &
\end{aligned}
$$

Of course, parameters $\alpha, k_{1}, k_{2}, k_{3}, k_{4}, k_{5}, k_{6}, k_{\mathrm{L}} \mathrm{a}, K_{\mathrm{b}}$, $K_{\mathrm{H}}, P_{\mathrm{T}}$, are supposed to be constant and known.

\section{A. Observability of $\mu_{1}$}

The differential polynomial, $P_{\mu_{1}}$, which introduces $\mu_{1}$ is too large to be reproduced here. It is available in the Appendix. A careful examination of this polynomial allows to see that it takes the form

$$
Q_{1} \dot{\mu}_{1}-\dot{Q}_{1} \mu_{1}-d_{1} Q_{1} \mu_{1}+Q_{1} \mu_{1}^{2}=0
$$

where

$$
Q_{1}=\left(q_{\mathrm{M}}+q_{\mathrm{C}}\right) a_{1}
$$


with

$$
\begin{aligned}
& a_{1}=k_{\mathrm{L}} \text { a } k_{6} K_{\mathrm{H}} P_{\mathrm{T}} D q_{\mathrm{M}} q_{\mathrm{C}}+k_{\mathrm{L}} \mathrm{a} k_{6} K_{\mathrm{H}} P_{\mathrm{T}} D q_{\mathrm{C}}^{2} \\
& +k_{\mathrm{L}} \text { a } k_{6} K_{\mathrm{H}} P_{\mathrm{T}} q_{\mathrm{M}} \dot{q}_{\mathrm{C}}-k_{\mathrm{L}} \text { a } k_{6} K_{\mathrm{H}} P_{\mathrm{T}} q_{\mathrm{C}} \dot{q}_{\mathrm{M}} \\
& +k_{\mathrm{L}} \text { a } k_{6} D Z_{\text {in }} q_{\mathrm{M}}^{2}+2 k_{\mathrm{L}} \text { a } k_{6} D Z_{\text {in }} q_{\mathrm{M}} q_{\mathrm{C}} \\
& +k_{\mathrm{L}} \text { a } k_{6} D Z_{\text {in }} q_{\mathrm{C}}^{2}-k_{\mathrm{L}} \text { a } k_{6} D C_{\text {in }} q_{\mathrm{M}}^{2} \\
& -2 k_{\mathrm{L}} \text { a } k_{6} D C_{\text {in }} q_{\mathrm{M}} q_{\mathrm{C}}-k_{\mathrm{L}} \text { a } k_{6} D C_{\text {in }} q_{\mathrm{C}}^{2} \\
& -k_{\mathrm{L}} \text { a } k_{6} D S_{2 \text { in }} q_{\mathrm{M}}^{2}-2 k_{\mathrm{L}} \text { a } k_{6} D S_{2 \text { in }} q_{\mathrm{M}} q_{\mathrm{C}} \\
& -k_{\mathrm{L}} \text { a } k_{6} D S_{2 \text { in }} q_{\mathrm{C}}^{2}+k_{\mathrm{L}} \text { a } k_{3} q_{\mathrm{M}}^{3}+2 k_{\mathrm{L}} \text { a } k_{3} q_{\mathrm{M}}^{2} q_{\mathrm{C}} \\
& +k_{\mathrm{L}} \text { a } k_{3} q_{\mathrm{M}} q_{\mathrm{C}}^{2}-k_{\mathrm{L}} \text { a } k_{5} q_{\mathrm{M}}^{3}-2 k_{\mathrm{L}} \text { a } k_{5} q_{\mathrm{M}}^{2} q_{\mathrm{C}} \\
& -k_{\mathrm{L}} \text { a } k_{5} q_{\mathrm{M}} q_{\mathrm{C}}^{2}+k_{\mathrm{L}} \text { a } k_{6} q_{\mathrm{M}}^{2} q_{\mathrm{C}}+2 k_{\mathrm{L}} \text { a } k_{6} q_{\mathrm{M}} q_{\mathrm{C}}^{2} \\
& +k_{\mathrm{L}} \text { a } k_{6} q_{\mathrm{C}}^{3}+k_{6} D q_{\mathrm{M}}^{2} q_{\mathrm{C}}+2 k_{6} D q_{\mathrm{M}} q_{\mathrm{C}}^{2} \\
& +k_{6} D q_{\mathrm{C}}^{3}+k_{6} q_{\mathrm{M}}^{2} \dot{q}_{\mathrm{C}}+2 k_{6} q_{\mathrm{M}} q_{\mathrm{C}} \dot{q}_{\mathrm{C}}+k_{6} q_{\mathrm{C}}^{2} \dot{q}_{\mathrm{C}}
\end{aligned}
$$

and

$$
d_{1}=\alpha D-\frac{\mathrm{d}}{\mathrm{d} t} \ln \left(\left(q_{\mathrm{M}}+q_{\mathrm{C}}\right)^{3}\right) .
$$

This says that $\mu_{1}$ is not observable (in the sense of [5]) with respect to $D, Z_{\text {in }}, S_{1 \text { in }}, S_{2 \text { in }}, C_{\text {in }}, q_{\mathrm{M}}, q_{\mathrm{C}}$ and $\mathrm{pH}$ since it is introduced by a differential equation of order 1 and not 0 .

But as previously explained in [4], dividing both sides of the previous equation by $\mu_{1}^{2}$, equation 2 becomes

$$
\dot{z}_{1}=-d_{1} z_{1}+Q_{1}
$$

with

$$
z_{1}=\frac{Q_{1}}{\mu_{1}} .
$$

If $d_{1}$ could be proved to be of constant positive sign then equation 4 would lead to an estimation scheme of $\mu_{1}$ with respect to $D, Z_{\mathrm{in}}, S_{1 \mathrm{in}}, S_{2 \mathrm{in}}, C_{\mathrm{in}}, q_{\mathrm{M}}, q_{\mathrm{C}}$ and $\mathrm{pH}$. The lack of clue on the positiveness of $d_{1}$ forces to look for a change of variable which is not as simple as in equation 5 .

Elementary but tedious manipulations of the differential polynomial $P_{\mu_{1}}$ allows to see that, if

$$
z_{1}=\frac{q_{1}}{\mu_{1}}
$$

with

$$
q_{1}=\frac{Q_{1}}{\left(q_{\mathrm{M}}+q_{\mathrm{C}}\right)^{3}}
$$

then equation 2 may be rewritten as

$$
\dot{z}_{1}=-\alpha D z_{1}+q_{1}
$$

which yields an estimation scheme for $\mu_{1}$ :

$$
\mu_{1}=\frac{q_{1}}{z_{1}}
$$

\section{B. Observability of $\mu_{2}$}

It happens that the differential polynomial introducing $\mu_{2}$ is much simpler than $P_{\mu_{1}}$, and is as follows:

$$
q_{\mathrm{M}} \dot{\mu}_{2}-\dot{q}_{\mathrm{M}} \mu_{2}-\alpha D q_{\mathrm{M}} \mu_{2}+q_{\mathrm{M}} \mu_{2}^{2}=0
$$

yielding

$$
\dot{z}_{2}=-\alpha D z_{2}+q_{\mathrm{M}}
$$

and

$$
\mu_{2}=\frac{q_{\mathrm{M}}}{z_{2}}
$$

\section{Design of the estimators}

Given their exponential stability (thanks to the constant positive sign of $\alpha D$ ) the differential equations 8 , and 11 are estimation schemes for $\mu_{1}$ and $\mu_{2}$. The speed of convergence of these estimators cannot be changed by the user, they are fixed by the quantity $\alpha D$.

In order to use equation 8 as an estimator for $\mu_{1}$ it is necessary to properly evaluate the quantity $q_{1}$ in equation 7 .

If the expression of $q_{1}$ taken from equations 7 and 3 were to be used as is in experimental data then difficulties would rise from the nonlinear dependency on measurements uncertainties through the time derivatives of $q_{\mathrm{M}}$ and $q_{\mathrm{C}}$.

Therefore the challenge is to reduce this complex expression of $q_{1}$ to a form which is more favorable to online numerical differentiation.

Again, elementary but subtle calculations allow to see that $q_{1}$ may be written as

$$
\begin{aligned}
q_{1}= & k_{\mathrm{L}} \mathrm{a} k_{6} K_{\mathrm{H}} P_{\mathrm{T}}\left(\frac{1}{1+\frac{q_{\mathrm{M}}}{q_{\mathrm{C}}}}\right)^{\bullet}+k_{6} \dot{q}_{\mathrm{C}} \\
& +k_{\mathrm{L}} \mathrm{a}\left(k_{3}-k_{5}\right) q_{\mathrm{M}}+k_{6}\left(k_{\mathrm{L}} \mathrm{a}+D\right) q_{\mathrm{C}} \\
& +k_{\mathrm{L}} \mathrm{a} k_{6} D\left(Z_{\mathrm{in}}-C_{\mathrm{in}}-S_{2 \mathrm{in}}\right)\left(\frac{1-\frac{q_{\mathrm{M}}}{q_{\mathrm{C}}}}{1+\frac{q_{\mathrm{M}}}{q_{\mathrm{C}}}}\right)^{2} \\
& +\frac{k_{\mathrm{L}} \mathrm{a} k_{6} K_{\mathrm{H}} P_{\mathrm{T}} D}{1+\frac{q_{\mathrm{M}}}{q_{\mathrm{C}}}} .
\end{aligned}
$$

Assuming the inputs $D, q_{1}$ and $q_{\mathrm{M}}$ free of $z_{i}$ the differential equations 8 , and 11 are readily dynamic estimators of $\mu_{1}$ and $\mu_{2}$ :

$$
\left\{\begin{array}{l}
\dot{\widehat{z}}_{i}=-\alpha D \widehat{z}_{i}+\widehat{y}_{i}, \\
\widehat{\mu}_{i}=\frac{\widehat{y}_{i}}{\widehat{z}_{i}},
\end{array}\right.
$$

where

$$
\widehat{y}_{1}=\widehat{q}_{1} \text { and } \widehat{y}_{2}=\bar{q}_{\mathrm{M}} \text {. }
$$

The quantities $\bar{q}_{\mathrm{C}}, \bar{q}_{\mathrm{M}}$ are the potentially noisy online measurements of $q_{\mathrm{C}}, q_{\mathrm{M}}$, respectively. There are two quantities which need to be numerically differentiated:

$$
\bar{q}_{\mathrm{C}} \text { and } \frac{1}{1+\frac{\bar{q}_{\mathrm{M}}}{\bar{q}_{\mathrm{C}}}} .
$$

The estimation error $\widetilde{z}_{i}=z_{i}-\widehat{z}_{i}$ evolves according to the following dynamics

$$
\dot{\widetilde{z}}_{i}=-\alpha D \widetilde{z}_{i}+\widetilde{y}_{i} \quad \text { with } \quad \widetilde{y}_{i}=y_{i}-\widehat{y}_{i} .
$$


In every time interval $[r, s]$, where the quantity $D$ is positive the estimation error decreases exponentially in norm as follows

$$
\begin{aligned}
\widetilde{z}_{i}(t)= & \widetilde{z}_{i}(r) \exp \left(-\int_{r}^{t} \alpha D(\sigma) \mathrm{d} \sigma\right) \\
& +\int_{r}^{t} \widetilde{y}_{i}(\sigma) \exp \left(-\int_{\sigma}^{t} \alpha D(\tau) \mathrm{d} \tau\right) \mathrm{d} \sigma
\end{aligned}
$$

when $t$ tends to $s$.

\section{A. Estimation scheme for $X_{1}$ and $S_{1}$}

The differential polynomial introducing $X_{1}$ reads as

$$
k_{\mathrm{L}} \text { a } k_{6}\left(k_{2}+k_{4}\right)\left(q_{\mathrm{M}}+q_{\mathrm{C}}\right)^{2} \mu_{1} X_{1}=a_{1}
$$

which leads to the following estimation of $X_{1}$ :

$$
\widehat{X}_{1}=\frac{\widehat{z}_{1}}{k_{\mathrm{L}} \mathrm{a}\left(k_{2}+k_{4}\right) k_{6}}
$$

where $z_{1}$ is given by equation 6 .

The differential equation for $S_{1}$ in equation 1 yields an estimator for this quantity:

$$
\dot{\widehat{S}}_{1}=-D \widehat{S}_{1}+D S_{1 \text { in }}-\frac{k_{1} \widehat{q}_{1}}{k_{\mathrm{L}} \mathrm{a}\left(k_{2}+k_{4}\right) k_{6}} .
$$

\section{B. Estimation scheme for $X_{2}$ and $S_{2}$}

The quantity $X_{2}$ is readily seen as

$$
\widehat{X}_{2}=\frac{\widehat{z}_{2}}{k_{6}}
$$

In addition, $S_{2}$ turns out to be able to be estimated as

$$
\widehat{S}_{2}=\widehat{Z}-\frac{K_{\mathrm{b}} K_{\mathrm{H}} P_{\mathrm{T}}}{\mathrm{pHe}} \frac{1}{1+\frac{\bar{q}_{\mathrm{M}}}{\bar{q}_{\mathrm{C}}}}-\frac{K_{\mathrm{b}} \bar{q}_{\mathrm{C}}}{k_{\mathrm{L}} \mathrm{a} \mathrm{pHe}},
$$

where $Z$ is given by

$$
\dot{\widehat{Z}}=-D \widehat{Z}+D Z i n
$$

\section{CONClusion}

It has been shown using the differential algebraic approach of observability and its accompanying differential algebraic decision methods (namely, characteristic set computations) that specific growth rates of the anaerobic wastewater of raw industrial wine distillery vinasses may be estimated from supposedly easily online measured data. This saves the time of empirical modeling and identification of specific growth rates, and allows the use of different kind of wastes without re-doing this empirical modeling. Moreover, it is believed that these estimates may serve as valuable tools in control and monitoring of highly unstable wastewater treatment processes.

\section{APPENDIX}

$P_{\mu_{1}}=\left(k_{\mathrm{L}}\right.$ a $k_{6} K_{\mathrm{H}} P_{\mathrm{T}} D q_{\mathrm{M}}^{2} q_{\mathrm{C}}+2 k_{\mathrm{L}}$ a $k_{6} K_{\mathrm{H}} P_{\mathrm{T}} D q_{\mathrm{M}} q_{\mathrm{C}}^{2}+$ $k_{\mathrm{L}} \mathrm{a}_{6} K_{\mathrm{H}} P_{\mathrm{T}} D q_{\mathrm{C}}^{3}+k_{\mathrm{L}} \mathrm{a} k_{6} K_{\mathrm{H}} P_{\mathrm{T}} q_{\mathrm{M}}^{2} \dot{q}_{\mathrm{C}} \quad-$ $k_{\mathrm{L}} \mathrm{a} k_{6} K_{\mathrm{H}} P_{\mathrm{T}} q_{\mathrm{M}} q_{\mathrm{C}} \dot{q}_{\mathrm{M}}+k_{\mathrm{L}}$ a $k_{6} K_{\mathrm{H}} P_{\mathrm{T}} q_{\mathrm{M}} q_{\mathrm{C}} \dot{q}_{\mathrm{C}}$ $k_{\mathrm{L}}$ a $k_{6} K_{\mathrm{H}} P_{\mathrm{T}} q_{\mathrm{C}}^{2} \dot{q}_{\mathrm{M}}+k_{\mathrm{L}}$ a $k_{6} D Z_{\mathrm{in}} q_{\mathrm{M}}^{3}$ $3 k_{\mathrm{L}}$ a $k_{6} D Z_{\mathrm{in}} q_{\mathrm{M}}^{2} q_{\mathrm{C}}+3 k_{\mathrm{L}}$ a $k_{6} D Z_{\text {in }} q_{\mathrm{M}} q_{\mathrm{C}}^{2} \quad+$ $k_{\mathrm{L}}$ a $k_{6} D Z_{\text {in }} q_{\mathrm{C}}^{3}-k_{\mathrm{L}}$ a $k_{6} D C_{\text {in }} q_{\mathrm{M}}^{3}-3 k_{\mathrm{L}}$ a $k_{6} D C_{\text {in }} q_{\mathrm{M}}^{2} q_{\mathrm{C}}-$ $3 k_{\mathrm{L}}$ a $k_{6} D C_{\text {in }} q_{\mathrm{M}} q_{\mathrm{C}}^{2}-k_{\mathrm{L}}$ a $k_{6} D C_{\text {in }} q_{\mathrm{C}}^{3}-k_{\mathrm{L}}$ a $k_{6} D S_{2 \text { in }} q_{\mathrm{M}}^{3}-$ $3 k_{\mathrm{L}}$ a $k_{6} D S_{2 \text { in }} q_{\mathrm{M}}^{2} q_{\mathrm{C}}-3 k_{\mathrm{L}}$ a $k_{6} D S_{2 \text { in }} q_{\mathrm{M}} q_{\mathrm{C}}^{2}-$ $k_{\mathrm{L}}$ a $k_{6} D S_{2 \text { in }} q_{\mathrm{C}}^{3}+k_{\mathrm{L}}$ a $k_{3} q_{\mathrm{M}}^{4}+3 k_{\mathrm{L}}$ a $k_{3} q_{\mathrm{M}}^{3} q_{\mathrm{C}}+$ $3 k_{\mathrm{L}}$ a $k_{3} q_{\mathrm{M}}^{2} q_{\mathrm{C}}^{2}+k_{\mathrm{L}}$ a $k_{3} q_{\mathrm{M}} q_{\mathrm{C}}^{3}-k_{\mathrm{L}}$ a $k_{5} q_{\mathrm{M}}^{4}-3 k_{\mathrm{L}}$ a $k_{5} q_{\mathrm{M}}^{3} q_{\mathrm{C}}-$ $3 k_{\mathrm{L}}$ a $k_{5} q_{\mathrm{M}}^{2} q_{\mathrm{C}}^{2}-k_{\mathrm{L}} \mathrm{a} k_{5} q_{\mathrm{M}} q_{\mathrm{C}}^{3}+k_{\mathrm{L}} \mathrm{a} k_{6} q_{\mathrm{M}}^{3} q_{\mathrm{C}}+$ $3 k_{\mathrm{L}}$ a $k_{6} q_{\mathrm{M}}^{2} q_{\mathrm{C}}^{2}+3 k_{\mathrm{L}}$ a $k_{6} q_{\mathrm{M}} q_{\mathrm{C}}^{3}+k_{\mathrm{L}} \mathrm{a} k_{6} q_{\mathrm{C}}^{4}+$ $k_{6} D q_{\mathrm{M}}^{3} q_{\mathrm{C}}+3 k_{6} D q_{\mathrm{M}}^{2} q_{\mathrm{C}}^{2}+3 k_{6} D q_{\mathrm{M}} q_{\mathrm{C}}^{3}+k_{6} D q_{\mathrm{C}}^{4}+$ $\left.k_{6} q_{\mathrm{M}}^{3} \dot{q}_{\mathrm{C}}+3 k_{6} q_{\mathrm{M}}^{2} q_{\mathrm{C}} \dot{q}_{\mathrm{C}}+3 k_{6} q_{\mathrm{M}} q_{\mathrm{C}}^{2} \dot{q}_{\mathrm{C}}+k_{6} q_{\mathrm{C}}^{3} \dot{q}_{\mathrm{C}}\right) \mu_{1}^{2}+$ $\left(-\alpha k_{\mathrm{L}}\right.$ a $k_{6} K_{\mathrm{H}} P_{\mathrm{T}} D^{2} q_{\mathrm{M}}^{2} q_{\mathrm{C}}-2 \alpha k_{\mathrm{L}}$ a $k_{6} K_{\mathrm{H}} P_{\mathrm{T}} D^{2} q_{\mathrm{M}} q_{\mathrm{C}}^{2}-$ $\alpha k_{\mathrm{L}}$ a $k_{6} K_{\mathrm{H}} P_{\mathrm{T}} D^{2} q_{\mathrm{C}}^{3}-\alpha k_{\mathrm{L}} \mathrm{a} k_{6} K_{\mathrm{H}} P_{\mathrm{T}} D q_{\mathrm{M}}^{2} \dot{q}_{\mathrm{C}}+$ $\alpha k_{\mathrm{L}}$ a $k_{6} K_{\mathrm{H}} P_{\mathrm{T}} D q_{\mathrm{M}} q_{\mathrm{C}} \dot{q}_{\mathrm{M}}-\alpha k_{\mathrm{L}}$ a $k_{6} K_{\mathrm{H}} P_{\mathrm{T}} D q_{\mathrm{M}} q_{\mathrm{C}} \dot{q}_{\mathrm{C}}+$ $\alpha k_{\mathrm{L}}$ a $k_{6} K_{\mathrm{H}} P_{\mathrm{T}} D q_{\mathrm{C}}^{2} \dot{q}_{\mathrm{M}}-\alpha k_{\mathrm{L}}$ a $k_{6} D^{2} Z_{\mathrm{in}} q_{\mathrm{M}}^{3}-$ $3 \alpha k_{\mathrm{L}}$ a $k_{6} D^{2} Z_{\text {in }} q_{\mathrm{M}}^{2} q_{\mathrm{C}}-3 \alpha k_{\mathrm{L}}$ a $k_{6} D^{2} Z_{\text {in }} q_{\mathrm{M}} q_{\mathrm{C}}^{2}-$ $\alpha k_{\mathrm{L}}$ a $k_{6} D^{2} Z_{\text {in }} q_{\mathrm{C}}^{3}+\alpha k_{\mathrm{L}}$ a $k_{6} D^{2} C_{\text {in }} q_{\mathrm{M}}^{3}+$ $3 \alpha k_{\mathrm{L}}$ a $k_{6} D^{2} C_{\text {in }} q_{\mathrm{M}}^{2} q_{\mathrm{C}}+3 \alpha k_{\mathrm{L}}$ a $k_{6} D^{2} C_{\text {in }} q_{\mathrm{M}} q_{\mathrm{C}}^{2}+$ $\alpha k_{\mathrm{L}}$ a $k_{6} D^{2} C_{\mathrm{in}} q_{\mathrm{C}}^{3}+\alpha k_{\mathrm{L}}$ a $k_{6} D^{2} S_{2 \text { in }} q_{\mathrm{M}}^{3}+$ $3 \alpha k_{\mathrm{L}}$ a $k_{6} D^{2} S_{2 \text { in }} q_{\mathrm{M}}^{2} q_{\mathrm{C}}+3 \alpha k_{\mathrm{L}}$ a $k_{6} D^{2} S_{2 \text { in }} q_{\mathrm{M}} q_{\mathrm{C}}^{2}+$ $\alpha k_{\mathrm{L}}$ a $k_{6} D^{2} S_{2 \text { in }} q_{\mathrm{C}}^{3}-\alpha k_{\mathrm{L}}$ a $k_{3} D q_{\mathrm{M}}^{4}-3 \alpha k_{\mathrm{L}}$ a $k_{3} D q_{\mathrm{M}}^{3} q_{\mathrm{C}}-$ $3 \alpha k_{\mathrm{L}}$ a $k_{3} D q_{\mathrm{M}}^{2} q_{\mathrm{C}}^{2}-\alpha k_{\mathrm{L}}$ a $k_{3} D q_{\mathrm{M}} q_{\mathrm{C}}^{3}+\alpha k_{\mathrm{L}}$ a $k_{5} D q_{\mathrm{M}}^{4}+$ $3 \alpha k_{\mathrm{L}} \mathrm{a} k_{5} D q_{\mathrm{M}}^{3} q_{\mathrm{C}}+3 \alpha k_{\mathrm{L}} \mathrm{a} k_{5} D q_{\mathrm{M}}^{2} q_{\mathrm{C}}^{2}+$ $\alpha k_{\mathrm{L}}$ a $k_{5} D q_{\mathrm{M}} q_{\mathrm{C}}^{3}-\alpha k_{\mathrm{L}}$ a $k_{6} D q_{\mathrm{M}}^{3} q_{\mathrm{C}}-3 \alpha k_{\mathrm{L}}$ a $k_{6} D q_{\mathrm{M}}^{2} q_{\mathrm{C}}^{2}-$ $3 \alpha k_{\mathrm{L}}$ a $k_{6} D q_{\mathrm{M}} q_{\mathrm{C}}^{3}-\alpha k_{\mathrm{L}}$ a $k_{6} D q_{\mathrm{C}}^{4}-\alpha k_{6} D^{2} q_{\mathrm{M}}^{3} q_{\mathrm{C}}-$ $3 \alpha k_{6} D^{2} q_{\mathrm{M}}^{2} q_{\mathrm{C}}^{2}-3 \alpha k_{6} D^{2} q_{\mathrm{M}} q_{\mathrm{C}}^{3}-\alpha k_{6} D^{2} q_{\mathrm{C}}^{4}-$ $k_{\mathrm{L}} \mathrm{a} k_{6} K_{\mathrm{H}} P_{\mathrm{T}} D q_{\mathrm{M}}^{2} \dot{q}_{\mathrm{C}}+k_{\mathrm{L}} \mathrm{a} k_{6} K_{\mathrm{H}} P_{\mathrm{T}} D q_{\mathrm{M}} q_{\mathrm{C}} \dot{q}_{\mathrm{M}}$ $k_{\mathrm{L}}$ a $k_{6} K_{\mathrm{H}} P_{\mathrm{T}} D q_{\mathrm{M}} q_{\mathrm{C}} \dot{q}_{\mathrm{C}}+k_{\mathrm{L}}$ a $k_{6} K_{\mathrm{H}} P_{\mathrm{T}} D q_{\mathrm{C}}^{2} \dot{q}_{\mathrm{M}}$ $k_{\mathrm{L}}$ a $k_{6} K_{\mathrm{H}} P_{\mathrm{T}} \dot{D} q_{\mathrm{M}}^{2} q_{\mathrm{C}}-2 k_{\mathrm{L}}$ a $k_{6} K_{\mathrm{H}} P_{\mathrm{T}} \dot{D} q_{\mathrm{M}} q_{\mathrm{C}}^{2}$ $k_{\mathrm{L}}$ a $k_{6} K_{\mathrm{H}} P_{\mathrm{T}} \dot{D} q_{\mathrm{C}}^{3}-\alpha k_{6} D q_{\mathrm{M}}^{3} \dot{q}_{\mathrm{C}}-3 \alpha k_{6} D q_{\mathrm{M}}^{2} q_{\mathrm{C}} \dot{q}_{\mathrm{C}}-$ $3 \alpha k_{6} D q_{\mathrm{M}} q_{\mathrm{C}}^{2} \dot{q}_{\mathrm{C}}-\alpha k_{6} D q_{\mathrm{C}}^{3} \dot{q}_{\mathrm{C}}-k_{\mathrm{L}} \mathrm{a} k_{6} K_{\mathrm{H}} P_{\mathrm{T}} q_{\mathrm{M}}^{2} \ddot{q}_{\mathrm{C}}+$ $k_{\mathrm{L}}$ a $k_{6} K_{\mathrm{H}} P_{\mathrm{T}} q_{\mathrm{M}} q_{\mathrm{C}} \ddot{q}_{\mathrm{M}} \quad-k_{\mathrm{L}}$ a $k_{6} K_{\mathrm{H}} P_{\mathrm{T}} q_{\mathrm{M}} q_{\mathrm{C}} \ddot{q}_{\mathrm{C}}+$ $2 k_{\mathrm{L}}$ a $k_{6} K_{\mathrm{H}} P_{\mathrm{T}} q_{\mathrm{M}} \dot{q}_{\mathrm{M}} \dot{q}_{\mathrm{C}}+2 k_{\mathrm{L}}$ a $k_{6} K_{\mathrm{H}} P_{\mathrm{T}} q_{\mathrm{M}} \dot{q}_{\mathrm{C}}^{2} \quad+$ $k_{\mathrm{L}} \mathrm{a} k_{6} K_{\mathrm{H}} P_{\mathrm{T}} q_{\mathrm{C}}^{2} \ddot{q}_{\mathrm{M}}-2 k_{\mathrm{L}} \mathrm{a} k_{6} K_{\mathrm{H}} P_{\mathrm{T}} q_{\mathrm{C}} \dot{q}_{\mathrm{M}}^{2}$ $2 k_{\mathrm{L}}$ a $k_{6} K_{\mathrm{H}} P_{\mathrm{T}} q_{\mathrm{C}} \dot{q}_{\mathrm{M}} \dot{q}_{\mathrm{C}} \quad-k_{\mathrm{L}}$ a $k_{6} D \dot{Z}_{\mathrm{in}} q_{\mathrm{M}}^{3}$ $3 k_{\mathrm{L}}$ a $k_{6} D \dot{Z}_{\text {in }} q_{\mathrm{M}}^{2} q_{\mathrm{C}}-3 k_{\mathrm{L}}$ a $k_{6} D \dot{Z}_{\text {in }} q_{\mathrm{M}} q_{\mathrm{C}}^{2}$ $k_{\mathrm{L}}$ a $k_{6} D \dot{Z}_{\mathrm{in}} q_{\mathrm{C}}^{3}+k_{\mathrm{L}}$ a $k_{6} D \dot{S}_{2 \text { in }} q_{\mathrm{M}}^{3}$ $3 k_{\mathrm{L}}$ a $k_{6} D \dot{S}_{2 \text { in }} q_{\mathrm{M}}^{2} q_{\mathrm{C}}+3 k_{\mathrm{L}}$ a $k_{6} D \dot{S}_{2 \text { in }} q_{\mathrm{M}} q_{\mathrm{C}}^{2} \quad+$ $k_{\mathrm{L}}$ a $k_{6} D \dot{S}_{2 \text { in }} q_{\mathrm{C}}^{3}-k_{\mathrm{L}}$ a $k_{6} Z_{\text {in }} \dot{D} q_{\mathrm{M}}^{3}-3 k_{\mathrm{L}}$ a $k_{6} Z_{\text {in }} \dot{D} q_{\mathrm{M}}^{2} q_{\mathrm{C}}-$ $3 k_{\mathrm{L}}$ a $k_{6} Z_{\text {in }} \dot{D} q_{\mathrm{M}} q_{\mathrm{C}}^{2}-k_{\mathrm{L}}$ a $k_{6} Z_{\text {in }} \dot{D} q_{\mathrm{C}}^{3}+$ $k_{\mathrm{L}}$ a $k_{6} C_{\mathrm{in}} \dot{D} q_{\mathrm{M}}^{3}+3 k_{\mathrm{L}}$ a $k_{6} C_{\mathrm{in}} \dot{D} q_{\mathrm{M}}^{2} q_{\mathrm{C}}+$ $3 k_{\mathrm{L}}$ a $k_{6} C_{\mathrm{in}} \dot{D} q_{\mathrm{M}} q_{\mathrm{C}}^{2} \quad+\quad k_{\mathrm{L}}$ a $k_{6} C_{\mathrm{in}} D q_{\mathrm{C}}^{3} \quad+$ $k_{\mathrm{L}}$ a $k_{6} S_{2 \text { in }} \dot{D} q_{\mathrm{M}}^{3}+3 k_{\mathrm{L}}$ a $k_{6} S_{2 \text { in }} \dot{D} q_{\mathrm{M}}^{2} q_{\mathrm{C}} \quad+$

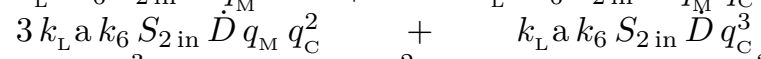
$k_{\mathrm{L}} \mathrm{a} k_{3} q_{\mathrm{M}}^{3} \dot{q}_{\mathrm{M}}-3 k_{\mathrm{L}}$ a $k_{3} q_{\mathrm{M}}^{2} q_{\mathrm{C}} \dot{q}_{\mathrm{M}}-3 k_{\mathrm{L}}$ a $k_{3} q_{\mathrm{M}} q_{\mathrm{C}}^{2} \dot{q}_{\mathrm{M}}-$ $k_{\mathrm{L}} \mathrm{a} k_{3} q_{\mathrm{C}}^{3} \dot{q}_{\mathrm{M}}+k_{\mathrm{L}} \mathrm{a} k_{5} q_{\mathrm{M}}^{3} \dot{q}_{\mathrm{M}}+3 k_{\mathrm{L}} \mathrm{a} k_{5} q_{\mathrm{M}}^{2} q_{\mathrm{C}} \dot{q}_{\mathrm{M}}+$ $3 k_{\mathrm{L}}$ a $k_{5} q_{\mathrm{M}} q_{\mathrm{C}}^{2} \dot{q}_{\mathrm{M}}+k_{\mathrm{L}} \mathrm{a} k_{5} q_{\mathrm{C}}^{3} \dot{q}_{\mathrm{M}}+\dot{C}_{\mathrm{in}} k_{\mathrm{L}} \mathrm{a} k_{6} D q_{\mathrm{M}}^{3}+$ $3 \dot{C}_{\mathrm{in}} k_{\mathrm{L}}$ a $k_{6} D q_{\mathrm{M}}^{2} q_{\mathrm{C}}+3 \dot{C}_{\mathrm{in}} k_{\mathrm{L}}$ a $k_{6} D q_{\mathrm{M}} q_{\mathrm{C}}^{2}+$ $\dot{C}_{\text {in }} k_{\mathrm{L}}$ a $k_{6} D q_{\mathrm{C}}^{3}-k_{\mathrm{L}}$ a $k_{6} q_{\mathrm{M}}^{3} \dot{q}_{\mathrm{C}}-3 k_{\mathrm{L}}$ a $k_{6} q_{\mathrm{M}}^{2} q_{\mathrm{C}} \dot{q}_{\mathrm{C}}-$ $3 k_{\mathrm{L}}$ a $k_{6} q_{\mathrm{M}} q_{\mathrm{C}}^{2} \dot{q}_{\mathrm{C}}-k_{\mathrm{L}} \mathrm{a} k_{6} q_{\mathrm{C}_{2}}^{3} \dot{q}_{\mathrm{C}}-k_{6} D q_{\mathrm{M}}^{3} \dot{q}_{\mathrm{C}}-$ $3 k_{6} D q_{\mathrm{M}}^{2} q_{\mathrm{C}} \dot{q}_{\mathrm{C}}-3 k_{6} D q_{\mathrm{M}} q_{\mathrm{C}}^{2} \dot{q}_{\mathrm{C}}-k_{6} D q_{\mathrm{C}}^{3} \dot{q}_{\mathrm{C}}-$ $k_{6} \dot{D} q_{\mathrm{M}}^{3} q_{\mathrm{C}}-3 k_{6} \dot{D} q_{\mathrm{M}}^{2} q_{\mathrm{C}}^{2}-3 k_{6} \dot{D} q_{\mathrm{M}} q_{\mathrm{C}}^{3}-k_{6} \dot{D} q_{\mathrm{C}}^{4}-$ 
$\left.k_{6} q_{\mathrm{M}}^{3} \ddot{q}_{\mathrm{C}}-3 k_{6} q_{\mathrm{M}}^{2} q_{\mathrm{C}} \ddot{q}_{\mathrm{C}}-3 k_{6} q_{\mathrm{M}} q_{\mathrm{C}}^{2} \ddot{q}_{\mathrm{C}}-k_{6} q_{\mathrm{C}}^{3} \ddot{q}_{\mathrm{C}}\right) \mu_{1}+$ $\left(k_{\mathrm{L}} \mathrm{a} k_{6} K_{\mathrm{H}} P_{\mathrm{T}} D q_{\mathrm{M}}^{2} q_{\mathrm{C}}+2 k_{\mathrm{L}}\right.$ a $k_{6} K_{\mathrm{H}} P_{\mathrm{T}} D q_{\mathrm{M}} q_{\mathrm{C}}^{2}+$ $k_{\mathrm{L}} \mathrm{a} k_{6} K_{\mathrm{H}} P_{\mathrm{T}} D q_{\mathrm{C}}^{3}+k_{\mathrm{L}} \mathrm{a} k_{6} K_{\mathrm{H}} P_{\mathrm{T}} q_{\mathrm{M}}^{2} \dot{q}_{\mathrm{C}}-$ $k_{\mathrm{L}}$ a $k_{6} K_{\mathrm{H}} P_{\mathrm{T}} q_{\mathrm{M}} q_{\mathrm{C}} \dot{q}_{\mathrm{M}}+k_{\mathrm{L}}$ a $k_{6} K_{\mathrm{H}} P_{\mathrm{T}} q_{\mathrm{M}} q_{\mathrm{C}} \dot{q}_{\mathrm{C}} \quad-$ $k_{\mathrm{L}}$ a $k_{6} K_{\mathrm{H}} P_{\mathrm{T}} q_{\mathrm{C}}^{2} \dot{q}_{\mathrm{M}}+k_{\mathrm{L}}$ a $k_{6} D Z_{\mathrm{in}} q_{\mathrm{M}}^{3} \quad+$ $3 k_{\mathrm{L}}$ a $k_{6} D Z_{\text {in }} q_{\mathrm{M}}^{2} q_{\mathrm{C}}+3 k_{\mathrm{L}}$ a $k_{6} D Z_{\text {in }} q_{\mathrm{M}} q_{\mathrm{C}}^{2} \quad+$ $k_{\mathrm{L}}$ a $k_{6} D Z_{\text {in }} q_{\mathrm{C}}^{3}-k_{\mathrm{L}}$ a $k_{6} D C_{\text {in }} q_{\mathrm{M}}^{3}-3 k_{\mathrm{L}}$ a $k_{6} D C_{\text {in }} q_{\mathrm{M}}^{2} q_{\mathrm{C}}-$ $3 k_{\mathrm{L}}$ a $k_{6} D C_{\text {in }} q_{\mathrm{M}} q_{\mathrm{C}}^{2}-k_{\mathrm{L}}$ a $k_{6} D C_{\text {in }} q_{\mathrm{C}}^{3}-k_{\mathrm{L}}$ a $k_{6} D S_{2 \text { in }} q_{\mathrm{M}}^{3}-$ $3 k_{\mathrm{L}}$ a $k_{6} D S_{2 \text { in }} q_{\mathrm{M}}^{2} q_{\mathrm{C}}-3 k_{\mathrm{L}}$ a $k_{6} D S_{2 \text { in }} q_{\mathrm{M}} q_{\mathrm{C}}^{2}-$ $k_{\mathrm{L}} \mathrm{a} k_{6} D S_{2 \text { in }} q_{\mathrm{C}}^{3}+k_{\mathrm{L}} \mathrm{a} k_{3} q_{\mathrm{M}}^{4}+3 k_{\mathrm{L}}$ a $k_{3} q_{\mathrm{M}}^{3} q_{\mathrm{C}}+$ $3 k_{\mathrm{L}}$ a $k_{3} q_{\mathrm{M}}^{2} q_{\mathrm{C}}^{2}+k_{\mathrm{L}}$ a $k_{3} q_{\mathrm{M}} q_{\mathrm{C}}^{3}-k_{\mathrm{L}}$ a $k_{5} q_{\mathrm{M}}^{4}-3 k_{\mathrm{L}}$ a $k_{5} q_{\mathrm{M}}^{3} q_{\mathrm{C}}-$ $3 k_{\mathrm{L}}$ a $k_{5} q_{\mathrm{M}}^{2} q_{\mathrm{C}}^{2}-k_{\mathrm{L}} \mathrm{a} k_{5} q_{\mathrm{M}} q_{\mathrm{C}}^{3}+k_{\mathrm{L}} \mathrm{a} k_{6} q_{\mathrm{M}}^{3} q_{\mathrm{C}}+$ $3 k_{\mathrm{L}}$ a $k_{6} q_{\mathrm{M}}^{2} q_{\mathrm{C}}^{2}+3 k_{\mathrm{L}}$ a $k_{6} q_{\mathrm{M}} q_{\mathrm{C}}^{3}+k_{\mathrm{L}}$ a $k_{6} q_{\mathrm{C}}^{4}+k_{6} D q_{\mathrm{M}}^{3} q_{\mathrm{C}}+$ $3 k_{6} D q_{\mathrm{M}}^{2} q_{\mathrm{C}}^{2}+3 k_{6} D q_{\mathrm{M}} q_{\mathrm{C}}^{3}+k_{6} D q_{\mathrm{C}}^{4}+k_{6} q_{\mathrm{M}}^{3} \dot{q}_{\mathrm{C}}+$ $\left.3 k_{6} q_{\mathrm{M}}^{2} q_{\mathrm{C}} \dot{q}_{\mathrm{C}}+3 k_{6} q_{\mathrm{M}} q_{\mathrm{C}}^{2} \dot{q}_{\mathrm{C}}+k_{6} q_{\mathrm{C}}^{3} \dot{q}_{\mathrm{C}}\right) \dot{\mu}_{1}$

\section{REFERENCES}

[1] V. Alcaraz-González, J. Harmand, A. Rapaport, J. P. Steyer, V. González-Alvarez, and C. Pelayo-Ortiz, Software sensors for highly uncertain WWTPs: a new approach based on interval observers, Water Research, 36(2002), 2515-2524, DOI: 10.1016/S0043-1354(01)00466-3.

[2] O. Bernard, Z. Hadj-Sadok, and D. Dochain, Software sensors to monitor the dynamics of microbial communities: Application to anaerobic digestion, Acta Biotheoretica, 48(2000), 197-205, DOI: 10 . 1023/A: 1010252725759.

[3] O. Bernard, Z. Hadj-Sadok, D. Dochain, A. Genovesi, and J. P. Steyer, Dynamic model development and parameter identification for an anaerobic wastewater treatment process, Biotechnology $\mathcal{G}$ Bioengineering, 75(2001), 424-438, DOI: 10.1002/bit. 10036.

[4] E. Chorukova, S. Diop, and I. Simeonov, On differential algebraic decision methods for the estimation of anaerobic digestion models, Algebraic Biology. Proc. Second Internat. Conf. ed. by H. Anai, K. Horimoto, and T. Kutsia, Lect. Notes Comput. Sci. vol. 4545, Springer-Verlag, Berlin-Heidelberg, 2007, pp. 202-216 DOI: 10 . 1007 / 978-3-540-734338_15.

[5] S. Diop, From the geometry to the algebra of nonlinear observability, Contemporary Trends in Nonlinear Geometric Control Theory and its Applications, ed. by A. Anzaldo-Meneses, B. Bonnard, J. P. Gauthier, and F. Monroy-Perez, World Scientific Publishing Co., Singapore, 2002, pp. 305-345 DOI: 10.1142/9789812778079_0012.

[6] S. Diop, On a differential algebraic approach of control observation problems, Algebraic and Algorithmic Aspects of Differential and Integral Operators Session '12 (LNCS post-proceedings), Lect. Notes Comput. Sci. Springer-Verlag, Berlin-Heidelberg, 2013,

[7] E. Petre, D. Selişteanu, and D. Şendrescu, Adaptive and robust-adaptive control strategies for anaerobic wastewater treatment bioprocesses, Chem. Engrg. Sci. 217(2013), 363-378, DOI: $10.1016 /$ j . cej . 2012.11.129. 